\title{
Diagnóstico e tratamento do hemangioma em lábio inferior: Relato de caso
}

\author{
Diagnosis and treatment of hemangioma in the lower lip: Case report \\ Diagnóstico y tratamiento del hemangioma en el labio inferior: Reporte de caso
}

Caroline Batista de Freitas ORCID: https://orcid.org/0000-0002-4769-7517 Centro Universitário Maurício de Nassau, Brasil E-mail: freitascaroline1@ hotmail.com

Ana Luiza Medeiros Cesar ORCID: https://orcid.org/0000-0003-2231-306X Universidade Federal Fluminense, Brasil E-mail: analumcesar@gmail.com

Felipe José Menezes Machado Santos ORCID: https://orcid.org/0000-0002-0571-8893 Universidade Tiradentes, Brasil

E-mail: felipemachado_santos@hotmail.com

Fernando Matheus Santana Tunel ORCID: https://orcid.org/0000-0001-7340-117X Universidade Tiradentes, Brasil E-mail: nandotunel@gmail.com

Gabriela Alves dos Santos

ORCID: https://orcid.org/0000-0001-5939-0953 Universidade Tiradentes, Brasil

E-mail: gabriela.alvess1@hotmail.com

Isadora de Andrade Souza

ORCID: https://orcid.org/0000-0003-0548-2777 Universidade Tiradentes, Brasil

E-mail: iisa_dora03@hotmail.com

Juliana Santos Almeida Costa

ORCID: https://orcid.org/0000-0002-0740-9189 Universidade Tiradentes, Brasil

E-mail: juliaana.almeida@oulook.com

Julyani Mota Souza Loeser

ORCID: https://orcid.org/0000-0002-8748-9456 Universidade Tiradentes, Brasil

E-mail: julyaniloeser@outlook.com

Layna Gabriely Muniz Santos ORCID: https://orcid.org/0000-0002-0302-0950 Universidade Tiradentes, Brasil

E-mail: laynagabrielly@hotmail.com Louise Cristina Santos

ORCID: https://orcid.org/0000-0001-7458-975X Universidade Tiradentes, Brasil

E-mail: louise_cristina123@hotmail.com

Mateus Ferreira Nogueira

ORCID: https://orcid.org/0000-0003-2417-1309

Universidade Tiradentes, Brasil

E-mail: mateusffnn@gmail.com

Maurício Santos Santana

ORCID: https://orcid.org/0000-0001-5340-4103 Universidade Tiradentes, Brasil

E-mail: mauriciosanttana10@gmail.com

Mônica Elisa Araújo Fernandes de Oliveira

ORCID: https://orcid.org/0000-0001-6767-9693 Universidade Tiradentes, Brasil

E-mail: monica_a.f@hotmail.com

Pedro Aragão de Souza

ORCID: https://orcid.org/0000-0002-9663-7449 Universidade Tiradentes, Brasil

E-mail: pedro_aragaos@hotmail.com

Pedro Vinícius Santos de Jesus

ORCID: https://orcid.org/0000-0001-9238-570X Universidade Tiradentes, Brasil E-mail: pedruk@hotmail.com 


\author{
Vanessa Gonçalves Moreira \\ ORCID: https://orcid.org/0000-0002-9471-0649 \\ Universidade Tiradentes, Brasil \\ E-mail: vanessagoncaalves@outlook.com \\ Vitória Virgínia Maria Machado Vanderley \\ ORCID: https://orcid.org/0000-0001-8972-3595 \\ Universidade Tiradentes, Brasil \\ E-mail: vitoriavirginiamachado@gmail.com \\ William José e Silva Filho \\ ORCID: https://orcid.org/0000-0002-2117-3352 \\ Universidade Federal de Sergipe, Brasil \\ E-mail: williamjsfilho10@gmail.com \\ Rafaella Bastos Leite \\ ORCID: https://orcid.org/0000-0002-3304-120X \\ Centro Universitário Maurício de Nassau, Brasil \\ E-mail: rrafaella_bastos@hotmail.com
}

\begin{abstract}
Resumo
O hemangioma é uma neoplasia benigna comum na região de cabeça e pescoço e é caracterizado por uma ploriferação anormal dos vasos sanguíneos, esta neoplasia tem predileção pelo sexo feminino e quando está associada as regiões oral e perioral, podem causar prejuízo estético e funcional a depender de sua localização. O objetivo desse estudo foi apresentar um caso clínico de hemangioma oral, discutir a importância do diagnóstico adequado bem como formas de tratamento para uma intervenção segura e cautelosa dessa patologia. Relato de caso: Paciente do sexo feminino, 54 anos, leucoderma, dona de casa, compareceu a clínica de Odontologia, com queixa principal de lesão enegrecida em lábio inferior e sem comprometimento sistêmico. Sob o procedimento de vitropressão, foi observado isquemia produzida pela placa de vidro e de acordo com a coleção sanguínea em seu interior, foi considerado a hipótese diagnóstica de hemangioma. Observando a natureza vascular do hemangioma foi feito a escolha de não realizar a biópsia devido a grande chance de ocorrer hemorragias. O tratamento realizado por meio de injeção da solução esclerozante de oleato de monoetanolamina promoveu a involução da lesão.
\end{abstract}

Palavras-chave: Malformações vasculares; Patologia bucal; Diagnóstico.

\begin{abstract}
Hemangioma is a benign neoplasm common in the head and neck region and is characterized by an abnormal ploriferation of blood vessels, this neoplasm has a predilection for females and when it is associated with the oral and perioral regions, they can cause aesthetic and functional damage depending on your location. The aim of this study was to present a clinical case of oral hemangioma, to discuss the importance of adequate diagnosis as well as ways of treatment for a safe and cautious intervention of this pathology. Case report: Female patient, 54 years old, leucoderma, housewife, attended the Dentistry clinic, with the main complaint of a blackened lesion on the lower lip and without systemic involvement. Under the procedure of vitro pressure, ischemia produced by the glass plate was observed and, according to the blood collection inside, the diagnostic hypothesis of hemangioma was considered. Observing the vascular nature of the hemangioma, the choice was made not to perform the biopsy due to the high chance of bleeding. The treatment performed by injection of the sclerosing solution of monoethanolamine oleate promoted the involution of the lesion.
\end{abstract}

Keywords: Vascular malformations; Pathology oral; Diagnosis.

\title{
Resumen
}

El hemangioma es una neoplasia benigna común en la región de la cabeza y el cuello y se caracteriza por una ploriferación anormal de los vasos sanguíneos. tu ubicación. El objetivo de este estudio fue presentar un caso clínico de hemangioma oral, para discutir la importancia de un diagnóstico adecuado, así como formas de tratamiento para una intervención segura y cautelosa de esta patología. Caso clínico: Paciente de sexo femenino, 54 años, leucodermia, ama de casa, acudió a la consulta de Odontología, con el principal síntoma de lesión ennegrecida en labio inferior y sin afectación sistémica. Bajo el procedimiento de vitropresión se observó isquemia producida por la placa de vidrio y, de acuerdo con la colección de sangre en su interior, se consideró la hipótesis diagnóstica de hemangioma. Al observar la naturaleza vascular del hemangioma, se optó por no realizar la biopsia por la alta probabilidad de sangrado. El tratamiento realizado mediante la inyección de la solución esclerosante de oleato de monoetanolamina favoreció la involución de la lesión.

Palabras clave: Malformaciones vasculares; Patología bucal; Diagnóstico.

\section{Introdução}

As anomalias vasculares são classificadas como lesões benignas dos vasos sanguíneos e são comuns nas regiões da 
cabeça e pescoço, incluindo a mucosa oral e lábios, as quais foram descritas de acordo com o sistema proposto por Milliken e Glowacki que pretendia estabelecer uma classificação biológica de hemangiomas e malformações vasculares fundamentada na apresentação clínica, comportamento biológico e histologia dessas lesões (Sales, 2015). O método descrito por eles atualmente é conhecido como o sistema oficial de classificação de doenças congênitas do desenvolvimento vascular (Costa, 2011; Guarniere, 2016).

O hemangioma é uma neoplasia benigna comum nas regiões da cabeça e pescoço, caracterizado por uma fase de crescimento proliferativo seguido por uma regressão inevitável muito lenta (fase involutiva) entre um e dez anos de idade, e sua permanência após os nove anos de idade é relativamente rara (Tolentino, 2020). Esta neoplasia tem predileção pelo sexo feminino e quando associada as regiões: oral e perioral, podem causar prejuízo estético e funcional a depender de sua localização. A região mais frequentemente acometida é o lábio, porém outras regiões como língua, mucosa jugal e palato também são referenciadas (Neves, 2018). Observa-se que a sua maior incidência é relatada logo após o nascimento ou na primeira infância; mas, alguns casos podem desenvolver-se na fase adulta. (Queiroz, 2014).

Em relação as características clínicas dessa neoplasia, apresentam-se como mancha ou nódulo arroxeada com conteúdo sangrento que desaparecem momentaneamente quando realizada a manobra da compressão digital ou diascopia, sua coloração varia do vermelho intenso ao roxo, de acordo com sua localização e profundidade do tecido (Neves, 2018). Histologicamente observa-se que as características celulares do hemangioma apresentam-se como células endoteliais imaturas, perocitos, células dendríticas, células mesenquimatosas com potencial adipogénico e uma pequena proporção de células germinativas (Silva, 2020).

A queixa mais prevalente entre os pacientes portadores de hemangioma é a questão estética, apesar de não serem alterações malignas os hemangiomas devem ser tratados, dependendo do tamanho e da localização, pois podem causar assimetria facial e a interferência na função mastigatória quando está presente em área susceptível a trauma pode ocorrer o risco de hemorragias, quando localizado na região de assoalho da boca, essas lesões causam problemas funcionais, associados à mastigação, deglutição e fonação além de afetar a qualidade de vida do paciente. (Palma, 2016).

O estabelecimento de um diagnóstico preciso é essencial para que seja possível identificar com clareza as distinções do hemangioma em relação as outras hipóteses diagnósticas que fazem diagnóstico diferencial com essa neoplasia, como por exemplo: mucocele, máculas, malformações vasculares e de outros tumores vasculares da infância. Nesse caso o diagnóstico diferencial pode ser estabelecido de forma simples e segura por meio da anamnese, exame clinico e por manobras semiotécnicas, como a vitropressão, que é uma técnica muito eficaz no estabelecimento do diagnóstico pois quando é realizada a compressão pela lâmina de vidro observa-se que a lesão adquire coloração pálida, essa situação ocorre em consequência da diminuição de tamanho e do esvaziamento vascular, após a remoção da pressão, o sangue retorna para o interior dos vasos e recupera sua aparência original (Silva, 2014; Fernandes, 2018).

Os tratamentos dos hemangiomas são controversos e irão depender da experiência e disponibilidade de recursos disponíveis, podendo ser realizado de diversas maneiras, dependendo de sua extensão e localização. Para as lesões menores e periféricas pode-se optar por esclerose química, excisão cirúrgica convencional, laserterapia, radioterapia, eletrocoagulação e crioterapia. Por outro lado, nas lesões maiores e/ ou intraósseas, o tratamento deve ser realizado através de embolização ou obliteração da lesão e dos vasos adjacentes (Sales, 2015).

Portanto, a proposta deste estudo será relatar um caso clínico de hemangioma oral tratado com oleato de etanolamina buscamos analisar e discutir os métodos de diagnóstico e as principais formas de tratamento do hemangioma oral, através de um protocolo simples e seguro, tendo em vista contribuir na escolha do tratamento adequado, na prática clínica dos profissionais e melhor prognóstico para os pacientes acometidos. 


\section{Relato de Caso}

Paciente do sexo feminino, 54 anos, leucoderma, compareceu a clínica de Odontologia, com queixa principal de lesão enegrecida em lábio inferior (Figura 1). Ao realizar o exame clínico extrabucal, foi observado uma pequena assimetria no lábio inferior direito, provocada pelo aumento de volume sem exibir nenhum comprometimento sistêmico. No exame clínico intraoral foi identificado na mucosa labial interna a presença de uma lesão de base séssil, com bordas bem delimitadas, medindo aproximadamente $2 \mathrm{~mm}$, localizada no lábio inferior direito e que se estendia para a mucosa labial interna, exibindo coloração arroxeada, com tempo de evolução de três anos. Sob o procedimento de vitropressão, foi observada isquemia produzida pela placa de vidro e de acordo com a coleção sanguínea em seu interior, foi considerado a hipótese diagnóstica de hemangioma oral (Figura 2).

Levando em consideração a interferência estética que esta patologia produz, optou-se pela terapia com o agente esclerozante, monoetanolamina, o qual age provocando a irritação da camada endotelial do vaso, proporcionando uma resposta inflamatória que leva a fibrose vascular. Foi realizada a utilização da monoetanolamina de $2 \mathrm{ml}$ em três aplicações, com concentrações de $1,25 \%$ de oleato de etanolamina com intervalo entre as sessões de uma semana, foi observado a regressão total da lesão, sem sinais de recidiva após cinco meses sob a terapia com este agente esclerosante. O presente relato de caso foi autorizado pelo paciente através da assinatura do Termo de Consentimento Livre e Esclarecido (TCLE).

Figura 1. Aspecto clínico inicial da má formação localizada em lábio inferior direito.

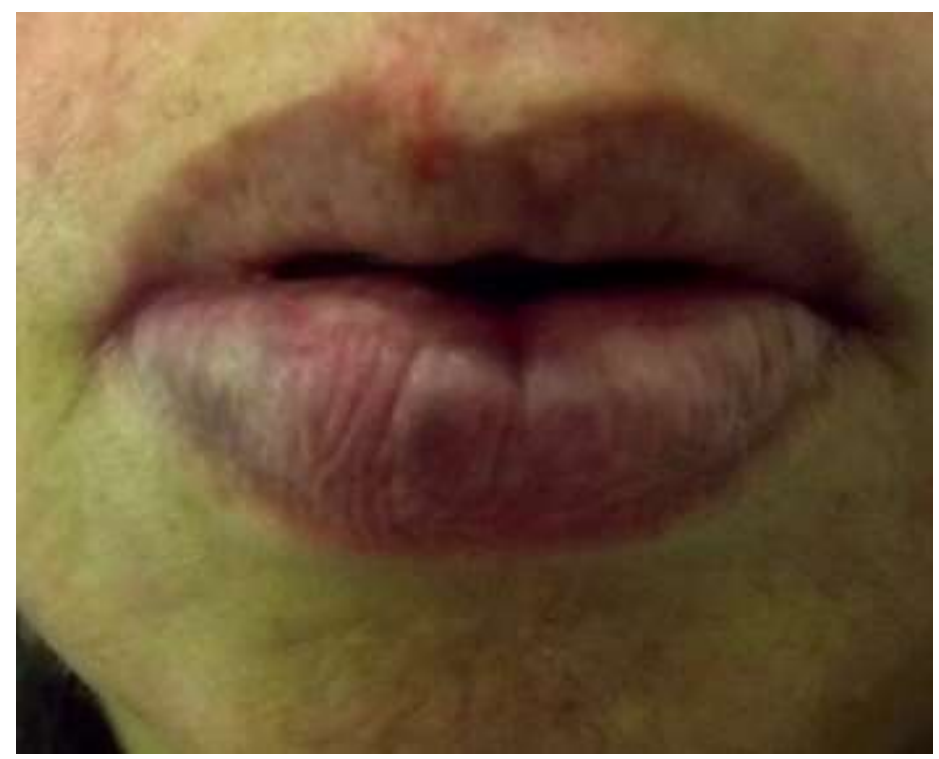

Fonte: Autores. 
Figura 2. Procedimento de vitropressão evidenciando a isquemia do lábio inferior.

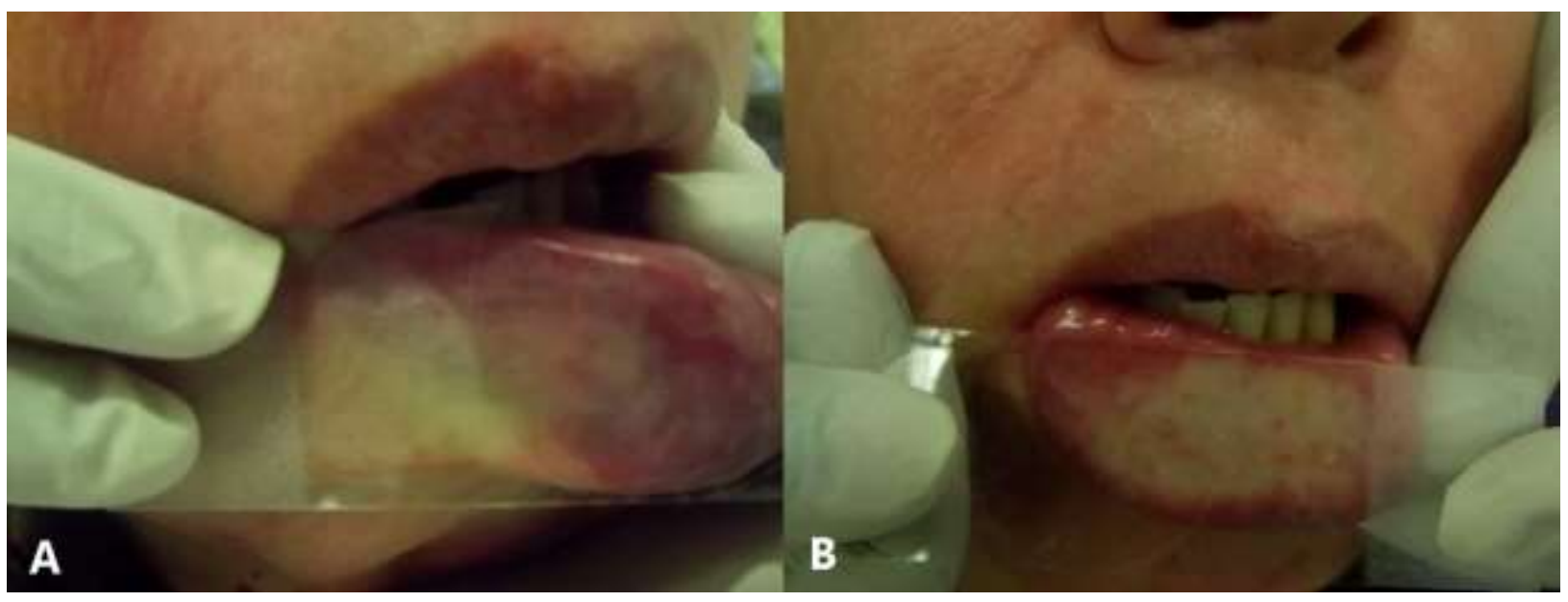

Fonte: Autores.

\section{Discussão}

De acordo com Neves (2018) o hemangioma é uma neoplasia benigna vascular e sua principal característica é a proliferação dos vasos sanguíneos. Essa lesão costuma acometer frequentemente a região do lábio, língua e mucosa jugal, geralmente apresenta-se como: mancha ou nódulo, circunscritos ou difusos, com conteúdo sangrento que desaparece momentaneamente quando realizada a manobra de compressão digital ou a diascopia, sua coloração varia do vermelho intenso ao roxo de acordo com sua localização e profundidade do tecido (Gomes, 2019). No caso descrito ao realizar o exame extra oral detectamos uma pequena assimetria no lábio inferior direito que não apresentava alterações importantes no aspecto extraoral.

Os hemangiomas podem ocorrer em qualquer fase da vida, sua etiologia está ligada a anomalias congênitas e traumas físicos, ainda de acordo com o relato de Neville ele descreve que essa patologia tem prevalência por pacientes do sexo feminino. No presente caso apresentado a paciente se encaixa no perfil descrito por Neville, por se tratar de uma paciente do sexo feminino, e apresentar 54 anos de idade. Na anamnese a paciente relatou o hábito de morder o lábio, porém este fato não existe relação com a etiologia do hemangioma, por se tratar de uma neoplasia (Silva, 2014; Fernandes, 2018).

Segundo Sales (2015) tratamentos dos hemangiomas são controversos e irão depender da experiência e disponibilidade de recursos disponíveis, podendo ser realizado de diversas maneiras, dependendo de sua extensão e localização, portanto o estabelecimento do diagnóstico é fundamental. No caso relatado, as características clínicas da lesão favoreceram o diagnóstico de hemangioma. A realização da manobra de vitropressão e a diascopia fez com que a lesão adquirisse uma coloração pálida, essa situação ocorreu em consequência da diminuição do tamanho e do esvaziamento vascular, distinguindo-as com clareza em relação a outras hipóteses diagnósticas já elencadas, a exemplo da mácula, mucocele e má formação vascular (Silva, 2014; Fernandes, 2018).

As lesões em face podem causar assimetria facial e acaba se tornando a queixa mais prevalente entre seus portadores durante a espera da involução da lesão, pois em alguns casos dependendo do tamanho e localização afeta diretamente a questão estética (Dantas, 2020). Diante disto, algumas terapias vêm sendo empregadas a fim de controlar o crescimento e antecipar a regressão do hemangioma. Para as lesões menores e periféricas pode-se optar por esclerose química, excisão cirúrgica convencional, laser terapia, radioterapia, eletrocoagulação e crioterapia, nas lesões maiores e/ou intraóssea o tratamento deve ser realizado através da embolização ou obliteração da lesão e dos vasos sanguíneos adjacentes (Sales, 2015).

Entre várias possibilidades terapêuticas, a esclerose por injeção de agente químico é uma das opções terapêuticas mais 
empregadas por apresentar grande vantagem, visto que o procedimento é pouco invasivo e não promove cicatriz aparente (Coimbra, 2020).

A escleroterapia é o método mais seguro, pois ela torna a possível regressão total ou parcial da lesão, em alguns casos a escleroterapia pode caracterizar o tratamento definitivo (Siqueira, 2020). No caso clínico relatado fizemos a utilização da monoetanolamina de $2 \mathrm{ml}$ em três aplicações com concentrações de 1,25\% de oleato de etanolamina, observamos a regressão total da lesão. Por a escleroterapia ser um procedimento minimamente invasivo e de acordo com a literatura propiciar a regressão total ou parcial da lesão e também apresentar excelentes resultados o presente trabalho optou pelo uso do oleato de etanolamina.

\section{Conclusão}

O hemangioma é uma patologia que acomete região de cabeça e pescoço, complexa e de difícil resolução se o diagnóstico e tratamento não forem bem estabelecidos e indicados. Existem muitas técnicas para o tratamento desta lesão a escleroterapia sem dúvidas é um tipo de tratamento viável, sendo muito eficaz e satisfatório. A injeção dessa substância promoveu a regressão total da lesão e favoreceu estética do paciente. Porém, cabe ressaltar que é necessário ter o conhecimento prévio da saúde do paciente, das indicações e contraindicações dessa técnica. Esse método deve ser cercado de todos os cuidados e avaliações necessárias como qualquer outro procedimento.

\section{Referências}

Costa, J. R. S. (2011). Sclerotherapy for Vascular Malformations in the Oral and Maxillofacial Region: Treatment and Follow-Up of 66 Lesions. J Oral Maxillofac Surg. 69: 88-89.

Dantas, F. S. B. (2020). Fotocoagulação a laser de diodo para tratamento de alteração vascular em lábio: relato de caso. Archives of health investigation. 9(5): 433-438.

Fernandes, D. (2018). Benign oral vascular lesions treated by sclerotherapy with ethalomine oleate: A retrospective study of 43 patients. Med. Oral Patol. Oral Cir. Bucal. 23: 181-185.

Gomes, J. A. (2019). Escleroterapia como tratamento conservador para hemangioma oral: relato de caso. Revista de Ciências Médicas e Biológicas. 18(3): 421-424.

Neves, L. (2018). Lesões vasculares orais: avaliações clínicas, diagnósticas e terapêuticas. Revista Cubana de Estomatologia. 55: 3-4.

Palma, F. (2016). Escleroterapia de hemangioma oral: relato de caso. Salusvita. 59: 85-87.

Queiroz, S. (2014). Tratamento de hemangioma oral com escleroterapia: relato de caso. J Vas Bras. 13: 250-251.

Sales, P. (2015). Utilização de agente esclerozante no tratamento de hemangioma língua: relato de caso. Rev. Odontol. Univ. Cid. São Paulo. $27: 254-255$.

Silva, W. B. (2014). Oral capillary hemangioma: A clinical protocol of diagnosis and treatment in adults. Oral Maxillofac Surg. 4: $231-237$.

Silva, E. L. C. (2020). Tratamento de hemangioma em mucosa labial por escleroterapia-Relato de caso clínico. Revista da Faculdade de Odontologia de Porto Alegre. 61(1): 115-122. 